\title{
Deformation-induced blueshift in emission spectrum of CdTe quantum dot composites
}

\author{
Pan Xiao ${ }^{1,3}$, Fujiu $\mathrm{Ke}^{2}$, Yilong Bai ${ }^{1}$ and Min Zhou ${ }^{\dagger 3}$ \\ ${ }^{1}$ LNM, Institute of Mechanics, Chinese Academy of Sciences, Beijing 100190, China \\ ${ }^{2}$ School of Physics and nuclear energy engineering, Beihang University, Beijing 100191, China \\ ${ }^{3}$ George W. Woodruff School of Mechanical Engineering, School of Materials Science and \\ Engineering, Georgia Institute of Technology, Atlanta, Georgia 30332-0405, USA
}

\section{ABSTRACT}

Polymer or glass films impregnated with quantum dots (QDs) have potential applications for mesoscale stress/strain sensing in the interior of materials under mechanical loading. One requirement in the development of such nanocomposite sensor materials is the establishment of calibrated relations between shifts in the emission spectrum of QD systems and the input stress/strain on the composites. Here, we use a multiscale computational framework to quantify the strain-dependent blueshift in the emission spectrum of CdTe QDs uniformly distributed in a matrix material under loading of a range of strain triaxiality. The framework, which combines the finite element method, molecular dynamics simulations and the empirical tight-binding method, captures the $\mathrm{QD} /$ matrix interactions, possible deformation-induced phase transformations and strain-dependent band structures of the QDs. Calculations reveal that the response of the QDs is strongly dependent on state of input strain. Under hydrostatic compression, the blueshift increases monotonically with strain. Under compression with lateral/axial strain ratios between

\footnotetext{
${ }^{\dagger}$ To whom correspondence should be addressed, Tel.: 404-894-3294, Fax: 404-894-0186, Email: min.zhou@gatech.edu
} 
0.0 and 0.5 , the blueshift initially increases, reaches a peak at an intermediate strain, and subsequently decreases with strain. This trend reflects a competition between increases in the energy levels associated with the conduction and valence bands of the QDs. The deformation-induced blueshift is also found to be dependent on QD orientations. The averaged blueshift over all orientations for the composite under uniaxial strain condition explains the blueshift variation trend observed in laser-driven shock compression experiments. Based on the simulation result, guidelines for developing QD composites as stress/strain sensing materials are discussed.

Keywords: CdTe quantum dot, band gap, empirical tight binding, molecular dynamics, finite element method, pressure sensor, laser-driven shock compression 


\section{Introduction}

Laser-driven shock compression (LSC) is a commonly used experimental technique to examine materials under loadings at extremely high strain rates (e.g., up to $10^{8} \mathrm{~s}^{-1}$ ) [1-5]. The LSC of microstructurally heterogeneous materials (materials composed of dissimilar constituents or materials containing geometric discontinuities such as voids, microcracks, interfaces or grain boundaries) usually results in highly inhomogeneous dynamic responses dominated by mesoscale processes in the interior of the materials which manifest on times scales of tens or hundreds of nanoseconds. Particle-level stress/strain measurement in the interior of materials under such extreme conditions requires special stress/strain sensing materials (SSM) that are small in size and fast in response. Specifically, the SSM should possess stress-dependent physical properties (e.g., optical properties) whose signal(s) can be captured and recorded in real time to achieve temporal resolutions of nanoseconds. Additionally, calibrated relations between the input stress/strain and the output optical responses of the SSM should be well established theoretically, computationally, and/or experimentally. Recently, a pressure-sensitive paint consisting of rhodamine 6G dye in poly-methylacryate (PMMA) polymer was found to be able to function as a recorder of the density profile under shock conditions with nanosecond resolutions $[6,7]$. However, due to the complex configuration of polymer, it is difficult to improve sensing accuracy via molecular design [7].

Quantum dots (QDs), with diameters of several nm, exhibit unique optical properties due to the quantum confinement effect [8]. They have been widely used as nanoscale electronic [9], photoluminescence [10,11], electrochemical [12] and temperature sensors [13]. QDs can also be used as pressure sensors and is potential candidates for stress/strain sensing. In particular, pressure- and strain-dependent optical properties have been reported for several types of QDs [14-16]. Compared 
polymer-based shock sensors (e.g., rhodamine 6G dye [7] and polyvinylidene fluoride polymer [17]) which are relatively large, QDs can be well controlled in experiments in terms of both size and shape. Since, QDs can be embedded in materials and optical emissions are used for measurement, techniques based on QDs are non-intrusive. Moreover, the optical responses of QDs under different loading condtions can be more precisely determined through theoretical analysis or numerical calculations [18].

Recently, LSC experiments on nanocomposite films on CdTe QDs dispersed in a soft polyvinyl alcohol (PVA) polymer matrix and in a hard inorganic sodium silicate glass matrix were carried out to study their suitability as internal SSM for LSC experiments $[19,20]$. Particle-level stress sensing with a temporal resolution of $0.5 \mathrm{~ns}$ was obtained in the experiments. The result points out that the wavelength blueshift history of QDs is deformation-dependent and can be used as an indicator and measure for internal stress during shock loading. It is found that the blueshift observed from LSC experiments first increases with pressure to a maximum and subsequently decreases with pressure. The trend is different from the monotonic increase of blueshift with pressure observed under conditions of quasistatic hydrostatic compression. Additionally, the blueshift in the LSC experiments is smaller than that in hydrostatic experiments at the same pressure levels. The difference highlights the need to delineate and understand the mechanism underlying the loading condition-dependent blueshift. The mulitscale nature of the structure of the QD composites and the coupling between the mechanical and optical properties necessitate a systematic analysis. Specifically, proper understanding and quantification of relations for the CdTe nanocomposite system should consider the deformation of the QDs in the matrix materials, the different stress/strain states in the constituents of the composite, the possibility of stress-induced phase transformations and the deformation-dependent nature of the band structures of the QDs. In the present work, a multiscale computational framework combining the empirical tight binding (ETB) method, molecular dynamics (MD) simulations and the finite element method (FEM) is employed to address this challenge. 
CdTe is a direct band gap semiconductor and the emission wavelengths of CdTe QDs are determined by their band structures. To calculate the band structure of a specific CdTe QD under a specific strain state, a quantum theory-based method is required. Several methods, such as the empirical pseudo-potential method $[21,22]$, ETB [23-25] and density functional theory [26] have been applied to calculate the strain-dependent band structures of QDs. The ETB method is used here due to its high efficiency and reliability [27]. Since phase transition was reported in bulk CdTe under high pressures [28-30], MD simulations are performed here to investigate the possibility of structure transformations of QDs under the conditions of interest. Our approach entails the use of top-down multiscale simulations. First, FEM simulations with explicit account of the QDs/matrix nanocomposite structure are carried out to quantify the stress/strain states of the QDs in the overall nanocomposite. The stress states so obtained are used as input boundary conditions for subsequent MD simulations. Finally, ETB calculations are used to calculate the band structures of the QDs which are in turn used to evaluate the emission spectrum and blueshift under given loading on the overall nanocomposite.

In experiments like those reported in [19] the effects of many factors, including stress-induced bandgap change, temperature, viscosity of the matrix materials, and charge of ligands at the $\mathrm{QD} /$ matrix interfaces, are at work. These effects cannot be experimentally isolated. Computational analyses can be used to isolate and analyze these effects. Such a task is carried out in this paper. Specifically, we focus on the effect of stress on the blueshift. Therefore, the band gap and blueshift of the QDs under loading of a range of strain triaxiality for the nanocomposite are considered. Since the QDs are distributed randomly in the matrix and the response of the QDs are orientation-dependent, the blueshift-strain relations for QDs in all possible spatial directions are used to evaluate the response of the QD ensemble as a whole under the conditions of different load triaxialities (or different stress states) of the overall nanocomposite. The results allow the differences in the trends of blueshift observed 
from the LSC and hydrostatic compression experiments to be explained. We conclude with considerations and guidelines for the development of CdTe QD nanocomposites as SSM in experiments.

\section{Computational Framework}

The composite film consisting of matrix material and uniformly distributed CdTe QDs is illustrated in Fig. 1(a). An illustration of a unit cell of the nanocomposite film is given in Fig. 1(b). The FEM model with element discretization for the unit cell used in the computations is shown in Fig. 1(c). Compression under conditions of uniaxial strain due to impact loading is in the vertical direction in Figs. 1(b-c). External pressure $\left(P_{z}\right)$ is applied on the top surface of the unit cell, with fixed constrains on the bottom surface. The lateral surfaces of the unit cell are constrained in the horizontal direction to effect the overall uniaxial state of strain under normal impact loading [20]. The silica glass [31] as used in experiments is the matrix material. To simplify the analysis, both CdTe and the glass matrix are assumed to be linear elastic. The specific material parameters are Young's modulus $(E)$, Poisson's ratio $(v)$ and mass density $(\rho)$, as listed in Table 1. In the experiments [20,32], the concentration $x_{Q D}$ of QDs in the matrix is approximately $0.15 \%$ by weight. For $x_{Q D}<<1$, the separation between QDs or the dimension of the unit cell $(L)$ can be estimated as

$$
L \approx\left(\frac{\pi \rho_{C d T e}}{6 \rho_{m} x_{Q D}}\right)^{1 / 3} d
$$

where $\rho_{m}$ and $\rho_{\text {CdTe }}$ are densities of the matrix and CdTe, respectively, and $d$ is the QD diameter. For QDs with diameters of 4.5 and $6.5 \mathrm{~nm}$, the estimated $L$ is about 43.9 and $63.4 \mathrm{~nm}$ (or 9.8d), respectively. 
The materials are represented by nodes and elements in FEM and by atoms in MD. In order to pass strain and deformation state information from FEM to MD, the MD simulations in the CdTe QD region of the unit cell uses a displacement boundary condition extracted from the FEM model. The implementation uses spatial interpolation of the displacement field at each atomic site, as illustrated in Fig. 2(c). After the application of the boundary displacement field, the deformed atomic configuration of the entire QD is relaxed with the surface atoms constrained via an equilibration MD calculation in order to ascertain if the applied deformation would lead to phase transformation in the QD. No phase transition is observed. A Stillinger-Weber (SW) type potential is used to describe the interatomic interactions in the CdTe atomic system [33]. The SW potential is known to provide reasonable tracking of different phases of CdTe [34]. Recently, a new analytical bond-order potential (BOP) was proposed for CdTe which gives some structural and properties trends close to those observed in experiments and quantum-mechanical calculations [34]. However, a good lattice constant is important for band structure calculations using the ETB method. Compared to the lattice constant of $6.48 \AA$ for the zinc-blende (ZB) structure of bulk CdTe measured in experiments, the lattice constant calculated from the BOP is $6.83 \AA$ which is not as good as the $6.51 \AA$ calculated from the SW potential. All the MD simulations are performed with LAMMPS [35], a molecular dynamics package developed by Sandia National Laboratories.

Since in MD and ETB methods the QD structure is represented by atoms, the relaxed atomic configuration of the $\mathrm{CdTe}$ QD at different strain states obtained from the MD calculations is used as input to the ETB method. In the ETB calculations, atomic positions are fixed to calculate band structures. The calculation of band structures is based on the empirical $\mathrm{sp}^{3} \mathrm{~s}^{*}$ model [36] including the spin-orbit interaction with parameters from Ref. [37]. With the same model and parameters, Pérez-Conde et al. [38] reported that the calculated size dependence of CdTe QD's energy gap shows reasonable agreement with available experimental data. The open source NEMO 3-D tool [27] which includes atomic strain effect due to its 
fundamental atomistic representations is employed to perform the ETB calculations. This tool has been widely used to calculate band structures of several types of QDs with different shapes under different strain conditions [39], especially for large-scale systems with millions of atoms.

The as-synthesized, spherically-shaped CdTe QDs are prepared by truncating the bulk ZB structure with the lattice constant $a=6.48 \AA$, as illustrated in Fig. 2(a). Surface atoms with single bonds are removed to avoid singularity. To study the size effects, two samples with QD diameters of $4.5 \mathrm{~nm}$ and $6.5 \mathrm{~nm}$ are considered. These diameters are smaller than the exciton Bohr radius of CdTe $(7.3 \mathrm{~nm})$, as shown in Fig. 2(b). Since CdTe is a direct band gap semiconductor, the band gap energy $\left(E_{g}\right)$ of a QD is calculated from the energy difference between the lowest unoccupied (LU) state of conduction bands and the highest occupied (HO) state of the valence bands, i.e., $E_{g}=E_{L U}-E_{H O}$. The calculated $E_{g}$ values are 1.85 and $1.74 \mathrm{eV}$ for relaxed 4.5 $\mathrm{nm}$ and $6.5 \mathrm{~nm}$ samples, respectively. These results agree well with available experimental data [38].

During experiments, the time stream of QD emission spectra is recorded $[6,20]$. Stress/strain sensitive information that can be extracted from the spectra includes emission intensity, average wavelength shift, and spectral width [7]. We focus on the strain-dependence of wavelength shift ( $\Delta \lambda)$ which can be determined from the band gap change of QDs via

$$
\Delta \lambda=h c\left(1 / E_{g}^{0}-1 / E_{g}^{d}\right),
$$

where $h$ is Planck's constant, $c$ is the speed of light, $E_{g}^{0}$ and $E_{g}^{d}$ are band gaps before and after deformation, respectively. Blueshift occurs if $\Delta \lambda>0$ and redshift occurs if $\Delta \lambda<0$. Unlike dyes which give redshift in polymers under shock compression [7], CdTe QDs primarily show blueshift under compressive loading. 


\section{Results and Discussion}

\subsection{Stress distribution in QD composites}

Fig. 3(a) shows the distributions of the normalized stress component in the loading direction $\left(\sigma_{z} / P_{z}\right)$ inside the unit cell with a $6.5 \mathrm{~nm}$ QD embedded in glass matrix. It can be seen that away from the QD in all directions, the stress decreases quickly to $P_{z}$ within a range of $2 d$. Therefore, interactions between the stress fields around individual QDs can be neglected when QD concentration $x_{Q D}$ is less than 0.15 wt $\%$ in experiment (or $L>9.8 d$ ). The FEM calculations allow QDs with different shapes and some non-linear attributes to be used for the analysis of QD and matrix interactions. However, for the simplified model here, an analytical solution can be obtained based on Eshelby's second (inhomogeneity) problem [40-42]. Specifically, the corresponding equivalent eigenstrain is [43]

$$
\varepsilon_{i j}^{* *}=\frac{15\left(\mu^{Q D}-\mu^{M}\right)\left(1-v^{M}\right)}{\left(5 v^{M}-7\right) \mu^{M}-\left(8-10 v^{M}\right) \mu^{Q D}} \varepsilon_{i j}^{0}+\frac{\left(K^{Q D}-K^{M}\right)\left(1-v^{M}\right)}{\left(4 v^{M}-2\right) K^{M}-\left(1+v^{M}\right) K^{Q D}} \varepsilon_{k k}^{0} \delta_{i j},
$$

where $\mu^{Q D}, \mu^{M}, K^{Q D}$ and $K^{M}$ are Lamé's second parameter and bulk modulus of the CdTe QDs and matrix material, respectively; and $\varepsilon_{i j}^{0}$ is the applied uniform strain field. According to Eshelby's theory, stress inside the QD is uniform

$$
\sigma_{i j}^{Q D}=C_{i j k l}^{Q D}\left(\varepsilon_{k l}^{0}+S_{k l m n} \varepsilon_{m n}^{* *}\right),
$$

where $C_{i j k l}^{Q D}$ is the stiffness matrix of the QD and $S_{k l m n}$ is Eshelby's tensor for spheres. In order to provide guidance for matrix material selection, the variation of the 
normalized stress component $\left(\sigma_{z} / P_{z}\right)$ inside the QD varies with the Young's modulus and Poisson's ratio of the matrix material is plotted in Fig. 3(b) using Eq. (2). The result reveals that, under the same external loading, matrices with lower Young's modulus or Poisson's ratio values lead to higher stress concentration inside the QD.

\subsection{Deformation-dependent blueshift of QDs}

The deformation of QDs in the overall uniaxial strain environment of the composite involves a strain ratio of $\gamma=\varepsilon_{r}^{Q D} / \varepsilon_{z}^{Q D}=-0.22$ (as determined from FEM calculations with the glass matrix), where $\varepsilon_{r}^{Q D}$ is the lateral strain in the $x y$ plane and $\varepsilon_{z}^{Q D}$ is the axial strain in the $z$ direction. Since shock compression of QD composite films results in highly heterogeneous internal deformation, a wide range of strain states are considered here. Specifically, states with $-0.5 \leq \gamma \leq 0$ and $\gamma=1$ are considered, with $\gamma=1$ denoting hydrostatic compression (strains in all directions are equal) and $\gamma=-0.5$ representing isochoric compression without volume change due to lateral expansion. This range of states is illustrated in Fig. 4. Since all cases considered involve compression in the $z$ direction, $\varepsilon_{r}^{Q D} \leq 0$. However, both compression and expansion in directions perpendicular to $z$ are considered, therefore, $\varepsilon_{r}^{Q D}$ can be both positive and negative. In general, the deformed shape of the QD is an ellipsoid. For the analysis carried out, $-0.1 \leq \varepsilon_{z}^{Q D} \leq 0$ and $\varepsilon_{r}^{Q D}=\gamma\left|\varepsilon_{z}^{Q D}\right|$.

Fig. 4 shows the blueshift of the $6.5 \mathrm{~nm}$ QDs as a function of $\varepsilon_{z}^{Q D}$ for the range of strain states considered. In all cases, the $z$ axis is along the [001] direction of the QDs. It can be seen that the blueshift under hydrostatic compression $(\gamma=1)$ increases with $\varepsilon_{z}^{Q D}$ monotonically with strain. This trend is similar to what is shown by 
previous numerical and experimental studies [44,45]. However, for $-0.5 \leq \gamma \leq 0$, the blueshiftinitially increases, reaches a peak (denoted as $\Delta \lambda_{\text {peak }}$ ) at an intermediate strain (denoted as $\varepsilon_{\text {peak }}^{Q D}$ ), and subsequently decreases with strain. For example, the blueshift for uniaxial strain $\gamma=-0.22$ reaches a peak value of $9.3 \mathrm{~nm}$ at $\varepsilon_{\text {peak }}^{Q D}=$ $2.6 \%$ and subsequently decreases with $\varepsilon_{z}^{Q D}$ to $0.0 \mathrm{~nm}$ at $\varepsilon_{z}^{Q D}=5.4 \%$ beyond which it becomes negative (redshift). Both $\Delta \lambda_{\text {peak }}$ and $\varepsilon_{\text {peak }}^{Q D}$ show dependence on $\gamma$. As $\gamma$ decrease from 0.0 to $-0.4, \Delta \lambda_{\text {peak }}$ decreases from 51.8 to $1.1 \mathrm{~nm}$ and $\varepsilon_{\text {peak }}^{Q D}$ decreases from $7.6 \%$ to $0.8 \%$. The results suggest that $\Delta \lambda_{\text {peak }}$ and $\varepsilon_{\text {peak }}^{Q D}$ are higher at higher levels of lateral confinement for the QDs. For $\gamma=-0.5$, only redshift occurs and no blueshift is observed.

Since MD simulations yielded no structure transitions, the existence of $\Delta \lambda_{\text {peak }}$ is not a result of any phase transformation. Rather, the blueshift is directly dependent on $E_{L U}$ and $E_{H O}$ which are plotted as functions of $\varepsilon_{z}^{Q D}$ in Fig. 5 for different values of $\gamma$. Under hydrostatic compression $(\gamma=1), E_{H O}$ decreases with strain, while $E_{L U}$ increases with strain, therefore, blueshift increases monotonically without a peak value. For $-0.5 \leq \gamma \leq 0$, both $E_{H O}$ and $E_{L U}$ increase with strain. However, $E_{L U}$ increases approximately linearly, while $E_{H O}$ increases nonlinearly. As a result, $E_{g}$ increases at small strains and decreases at larger strains, leading to the blueshift peak. Also, at the same strain, $E_{H O}$ increases with $\gamma$ while $E_{L U}$ shows an opposite trends, as a result, both $\Delta \lambda_{\text {peak }}$ and $\varepsilon_{\text {peak }}^{Q D}$ increase with $\gamma$. For $\gamma=-0.5$, $E_{L U}$ shows a very weak dependence on strain, while $E_{H O}$ increases with strain. As a result, $\gamma=-0.5$ always leads to redshift. 


\subsection{Orientation dependence of blueshift}

To study the orientation dependence of blueshift, we consider deformations with $\gamma=0.0$ and $\gamma=-0.22$ and $\varepsilon_{z}^{Q D}$ along different lattice directions. Fig. 6(a) shows the orientation-dependent blueshift of a QD for uniaxial strain $(\gamma=-0.22)$ with $\varepsilon_{z}^{Q D}$ along lattice directions ranging from the $[001]$ to the $[111]$ directions in the $(1 \overline{1} 0)$ plane. These directions are denoted by angle $\varphi$ relative to the $z$ axis, as illustrated in Fig. 6(b). The blueshift is strongly orientation-dependent and $\Delta \lambda_{\text {peak }}$ is observed for all orientations. The lowest is $\Delta \lambda_{\text {peak }}=9.3 \mathrm{~nm}$ for the [001] direction $\left(\varphi=0^{\circ}\right)$, and the highest is $\Delta \lambda_{\text {peak }}=49.3 \mathrm{~nm}$ for the [111] direction. Fig. 6(c) shows a polar view of the blueshift for both $\gamma=0.0$ and $\gamma=-0.22$ at a strain of $\varepsilon_{z}^{Q D}=0.06$ along directions in the $(1 \overline{1} 0)$ plane. The higher lateral confinement under $\gamma=0.0$ relative to that under $\gamma=-0.22$ causes the bluseshift for $\gamma=0.0$ to be higher for all the direction in the plane. However, the curves are similar, suggesting that straining along the [001] direction always results in the lowest blueshift regardless of confinement or strain state. The curve for $\gamma=-0.22$ shows larger variations from direction to direction, indicating that lateral confinement also causes the differences in response between directions to be smaller.

Since QDs are dispersed in the matrix with random orientations, their actual crystalline directions which coincide with the impact direction of a sample are also random and span all possible crystalline orientations. As a result, the emission spectra for such samples consist of contribution from responses from all possible crystalline orientations. To obtain the average wavelength shift which is used as a measure for internal states of stress/strain [6], contributions to blueshift from all orientations are 
averaged. Strictly speaking, due to the lattice structure of QDs, both mechanical properties (e.g., elasticity) and strain-induced blueshift are orientation-dependent. If both are considered at the same time, it would be difficult to perform the calculations. Additionally, the orientation-dependency of the strain-induced blueshift is much more pronounced than that of mechanical properties. Here, a simplified approach is taken, focusing on blueshift by using an isotropic elasticity model. To help visualize the orientation dependence of the blueshift, $\Delta \lambda^{(\varphi, \theta)}$ is used to denote the blueshift for the polar direction $(\varphi, \theta)$ in the polar coordinate system with the [001] direction as the polar axis. A numerical approach is taken in evaluating the average. To this end and because of symmetry, the response $\left(\Delta \lambda^{(\varphi, \theta)}\right)$ in a number of directions in the octant between the (100) and (1 $\overline{1} 0)$ planes (Fig. 6) are calculated and used to obtain the average. These directions are evenly spaced on the (100), (1 $\overline{1} 0)$ and $(2 \overline{1} 0)$ planes. Additionally, when a composite film is compressed in experiments, all embedded QDs are subjected to the same external stress $P_{z}$ in the loading direction. The average blueshift for $\gamma=-0.22$ so obtained is shown as a function of input stress $P_{z}$ in Fig. 7. The overall trend bears resemblance to that for individual orientations, except that the values of $\Delta \lambda_{\text {peak }}$ and $P_{\text {peak }}$ (the input stress at which $\Delta \lambda_{\text {peak }}$ occurs) are different. Specifically, for the $6.5 \mathrm{~nm}$ QDs, a $\Delta \lambda_{\text {peak }}=25.1 \mathrm{~nm}$ occurs at $P_{\text {peak }}=5.1 \mathrm{GPa}$; and for the $4.5 \mathrm{~nm}$ QDs $\Delta \lambda_{\text {peak }}=27.1 \mathrm{~nm}$ occurs at $P_{\text {peak }}$ $=5.3 \mathrm{GPa}$. Fig. 7 also shows 3-dimensinal views of the orientation-dependent blueshift for the $6.5 \mathrm{~nm}$ QDs at the same average blueshift level of $20.0 \mathrm{~nm}$ under different stress levels (A and B). Although the average blueshift is the same, the blueshift distributions are quite different. Compared to stress state A, state B shows a wider wavelength shift range with both blueshift and redshift (not shown in Fig. 7).

\subsection{Considerations for developing CdTe QD composite-based SSM}


It is worth noting that several factors in the experiments involving the CdTe QD composite [20] have not been considered in this paper, including (i) the molecular structure of the interfaces between the QDs and the matrix; (ii) full dynamic shock loading process and (iii) shock-induced temperature changes and their effects on light emission. These factors may lead to quantitative differences between experimental measurements and the calculated results and need to be investigated in the future. Nevertheless, the calculated results are in qualitative agreement with experimental observations, including the fact that non-hydrostatic strain states lead to non-monotonic changes of emission wave length with input stress [19]. Another finding is that the strain state, as measured by the ratio between the lateral strain and the axial strain, and the orientation of QDs profoundly affect the wavelength shift. In particular, lateral confinement can lead to lower blueshift relative to that under hydrostatic loading. The results here also provide the following insight and suggestions for the development of CdTe QD composite-based stress/strain sensors for LSC experiments.

Selection of QD size and matrix material: CdTe QDs composites with higher $\Delta \lambda_{\text {peak }}$ and $P_{\text {peak }}$ values are more desirable for stress sensing, because they offer monotonous response over wider ranges of input stress before reaching $\Delta \lambda_{\text {peak }}$. Higher blueshift levels also provide higher sensitivities for measurement. The emission wavelength of CdTe QD is size-dependent, yet simulations of $4.5 \mathrm{~nm}$ and $6.5 \mathrm{~nm}$ QDs do not show significant differences in the average blueshift, as seen in Fig. 7(a). For $\gamma=-0.22, \Delta \lambda_{\text {peak }}=25.1 \mathrm{~nm}$ for the $6.5 \mathrm{~nm}$ QDs, which is $7.6 \%$ lower than that of the $4.5 \mathrm{~nm}$ QDs, while $P_{\text {peak }}$ for the two cases differ by $7.3 \%$. On the other hand, $\gamma$ affects blueshift more significantly than the diameter of the QDs. Higher lateral confinement on QDs leads to higher $\Delta \lambda_{\text {peak }}$ and $P_{\text {peak }}$, suggesting that 
increasing the stiffness or Poisson ratio of the matrix material enhances the sensitivity and accuracy of the sensor material. Impact loading generates overall uniaxial compressive states of strain at the overall sample level, with the understanding that material heterogeneity and microstructure such as that of the QD nanocomposite considered can cause local conditions to deviate from the overall average. For the CdTe QD nanocomposite, $\gamma=0.0$ at the overall sample level and $\gamma=-0.22$ for individual QD particles. This value is dependent on matrix property and can be determined by Eq. (2) of Eshelby's theory.

Phase transformation: It is reported that bulk CdTe undergoes a phase transition under pressures of about 4.0 GPa [28,29,46]. Although no direct observation of phase transforms has been made for CdTe QDs under hydrostatic pressure, signatures in the measurements of several properties, including thermal diffusivity and Resonant Raman spectra [45,47], suggests that phase transitions may happen in CdTe QDs at about 5.8 GPa [48], just like in the bulk counterpart. In our simulations, the QDs show no phase transformation under strains lower than 10\%. Any pressure-induced phase transformation may lead to the loss of emission fluorescence or variation in blueshift $[48,49]$. However, our calculation suggests that there exists a deformation-induced $\Delta \lambda_{\text {peak }}$ before any phase transformation happens. In fact, in the LSC experiment [20], emission fluorescence is still observed for the CdTe QDs with shock input stresses up to $7.3 \mathrm{GPa}$. Therefore, we logically conclude that the blueshift trend of first increasing with input stress and subsequently decreasing after a critical value as observed in experiments can be attributed to deformation rather than phase transformation.

Determination of stress from emission spectra: In experiments, the time history of emission spectra can be obtained. Since there is a peak blueshift for non-hydrostatic compression, the relation between the average blueshift and input stress is multi-valued (Fig. 7), creating potential ambiguity in the determination of 
stress. To distinguish between possible stress states, the distribution of blueshift should be considered using the orientation dependence information in Fig. 7. However, this is not the only possible way to determine the logical choice among multiple possibilities. In actual experiments, the internal strain state can be determined by combining analyses of fluorescence intensity-loss, different moments of the emission spectrum $[7,28]$ and other related measures from experiments.

\section{Summary}

The strain-dependent optical response of $\mathrm{CdTe} \mathrm{QD} /$ matrix composites is investigated via a multiscale computational framework. The framework combines the FEM, MD and ETB methods, capturing matrix/QD interactions, possible deformation-induced phase transformations and strain-dependent band structures of the QDs. Calculations reveal that the response of the QDs is strongly dependent on their state of input stress. Under hydrostatic compression, the blueshift increases monotonically with strain. Under compressive deformation with lateral/axial strain ratios of $-0.5 \leq \gamma \leq 0$, the blueshift initially increases, reaches a peak $\left(\Delta \lambda_{\text {peak }}\right)$ at an intermediate strain, and subsequently decreases with strain. QDs subject to higher levels of lateral confinement (lower $\gamma$ ) show larger blueshift and $\Delta \lambda_{\text {peak }}$. Analyses of the band structure reveal that the existence of $\Delta \lambda_{\text {peak }}$ results from a competition between increases in $E_{L U}$ and $E_{H O}$ instead of phase transformation. The strain-induced blueshift is also found to be dependent on QD orientations. The largest and smallest blueshift are found to be associated with the [111] and [001] directions, respectively. The average blueshift over all orientations shows a trend consistent with observations in experiments. The blueshift-stress relations obtained from the calculations provide qualitative relations that should be further refined via experiments and account of more realistic factors. The calculations have also allowed insight to be gained regarding the selection of QD size and matrix material, possibility 
of phase transformations and determination of pressure from emission spectra.

\section{Acknowledgements}

The authors gratefully acknowledge support from the Defense Threat Reduction Agency (DTRA) [grant number HDTRA1-12-1-0052], the Strategic Priority Research Program (B) of the Chinese Academy of Sciences [grant number XDB22040501] and the National Natural Science Foundation of China [grant numbers 11202212, 11432014 and 11672298]. Calculations are carried out on parallel computers at DPRL at Georgia Tech, the ScGrid Supercomputing Center, and the Computer Network Information Center of the Chinese Academy of Sciences. 


\section{References}

[1] Brown KE, Shaw WL, Zheng X, Dlott DD. Simplified laser-driven flyer plates for shock compression science. Rev Sci Instrum 2012;83:103901. doi:10.1063/1.4754717.

[2] Trainor RJ, Shaner JW, Auerbach JM, Holmes NC. Ultrahigh-pressure laser-driven shock-wave experiments in aluminum. Phys Rev Lett 1979;42:1154-7. doi:10.1103/PhysRevLett.42.1154.

[3] Trott WM. Development of Laser-Driven Flyer Techniques for Equation-of-State Studies of Microscale Materials. AIP Conf. Proc., vol. 620, 2002, p. 1347-50. doi:10.1063/1.1483788.

[4] Paisley DL, Luo SN, Greenfield SR, Koskelo AC. Laser-launched flyer plate and confined laser ablation for shock wave loading: Validation and applications. Rev Sci Instrum 2008;79. doi:10.1063/1.2839399.

[5] Swift DC, Niemczura JG, Paisley DL, Johnson RP, Luo S-N, Tierney TE. Laser-launched flyer plates for shock physics experiments. Rev Sci Instrum 2005;76:93907. doi:10.1063/1.2052593.

[6] Banishev AA, Shaw WL, Dlott DD. Dynamics of polymer response to nanosecond shock compression. Appl Phys Lett 2014;104:101914. doi:10.1063/1.4868723.

[7] Banishev AA, Dlott DD. Ultrafast pressure-sensitive paint for shock compression spectroscopy. J Appl Phys 2014;115:203515. doi:10.1063/1.4880401.

[8] Chan WC. Quantum Dot Bioconjugates for Ultrasensitive Nonisotopic Detection. Science 1998;281:2016-8. doi:10.1126/science.281.5385.2016.

[9] Wang L-J, Cao G, Tu T, Li H-O, Zhou C, Hao X-J, et al. A graphene quantum dot with a single electron transistor as an integrated charge sensor. Appl Phys Lett 2010;97:262113. doi:10.1063/1.3533021.

[10] Murphy CJ. Optical sensing with quantum dots. Anal Chem 2002;74:520A-526A. doi:10.1021/ac022124v.

[11] Medintz IL, Uyeda HT, Goldman ER, Mattoussi H. Quantum dot bioconjugates for imaging, labelling and sensing. Nat Mater 2005;4:435-46. doi:10.1038/nmat1390.

[12] Sun H, Wu L, Wei W, Qu X. Recent advances in graphene quantum dots for sensing. Mater Today 2013;16:433-42. doi:10.1016/j.mattod.2013.10.020.

[13] Li S, Zhang K, Yang J-M, Lin L, Yang H. Single quantum dots as local temperature markers. Nano Lett 2007;7:3102-5. doi:10.1021/n1071606p.

[14] Ellaway SW, Faux DA. Effective elastic stiffnesses of InAs under uniform strain. J Appl Phys 2002;92:3027. doi:10.1063/1.1500421.

[15] Drickamer HG, Frank CW, Slichter CP. Optical Versus Thermal Transitions in Solids at High Pressure. Proc Natl Acad Sci 1972;69:933-7. doi:10.1073/pnas.69.4.933.

[16] Narayanan M, Peter AJ. Pressure and Temperature Induced Non-Linear Optical Properties in a 
Narrow Band Gap Quantum Dot. Quantum Matter 2012;1:53-8.

doi:doi:10.1166/qm.2012.1005.

[17] Neel C, Thadhani N. Shock and release wave speed of an alumina epoxy composite. J Appl Phys 2009;106:2007-10. doi:10.1063/1.3204468.

[18] Woggon U. Optical Properties of Semiconductor Quantum Dots. vol. 136. Berlin, Heidelberg: Springer Berlin Heidelberg; 1997. doi:10.1007/BFb0119351.

[19] Kang Z, Banishev AA, Lee G, Scripka DA, Breidenich J, Xiao P, et al. Exploration of CdTe quantum dots as mesoscale pressure sensors via time-resolved shock-compression photoluminescent emission spectroscopy. J Appl Phys 2016;120:43107. doi:10.1063/1.4959257.

[20] Xiao P, Kang Z, Bansihev AA, Breidenich J, Scripka DA, Christensen JM, et al. Laser-excited optical emission response of CdTe quantum dot/polymer nanocomposite under shock compression. Appl Phys Lett 2016;108:11908. doi:10.1063/1.4939701.

[21] Luo JW, Bester G, Zunger A. Atomistic pseudopotential calculations of thickness-fluctuation GaAs quantum dots. Phys Rev B - Condens Matter Mater Phys 2009;79:1-15. doi:10.1103/PhysRevB.79.125329.

[22] Williamson AJ, Wang LW, Zunger A. Theoretical interpretation of the experimental electronic structure of lens-shaped self-assembled InAs/GaAs quantum dots. Phys Rev B - Condens Matter Mater Phys 2000;62:12963-77. doi:10.1103/PhysRevB.62.12963.

[23] Zieliński M. Including strain in atomistic tight-binding Hamiltonians: An application to self-assembled InAs/GaAs and InAs/InP quantum dots. Phys Rev B - Condens Matter Mater Phys 2012;86:115424. doi:10.1103/PhysRevB.86.115424.

[24] Talwar DN, Ting CS. Tight-binding calculations for the electronic structure of isolated vacancies and impurities in III-V compound semiconductors. Phys Rev B 1982;25:2660-80. doi:10.1103/PhysRevB.25.2660.

[25] Santoprete R, Koiller B, Capaz RB, Kratzer P, Liu QKK, Scheffler M. Tight-binding study of the influence of the strain on the electronic properties of InAs/GaAs quantum dots. Phys Rev B 2003;68:235311. doi:10.1103/PhysRevB.68.235311.

[26] Cho E, Jang H, Lee J, Jang E. Modeling on the size dependent properties of InP quantum dots: a hybrid functional study. Nanotechnology 2013;24:215201. doi:10.1088/0957-4484/24/21/215201.

[27] Usman M, Ryu H, Woo I, Ebert DS, Klimeck G. Moving toward nano-TCAD through multimillion-atom quantum-dot simulations matching experimental data. IEEE Trans Nanotechnol 2009;8:330-44. doi:10.1109/TNANO.2008.2011900.

[28] Alptekin S. Structural phase transition of CdTe: An ab initio study. J Mol Model 2013;19:421-6. doi:10.1007/s00894-012-1568-z.

[29] McMahon MI, Nelmes RJ, Wright NG, Allan DR. Phase transitions in CdTe to 5 GPa. Phys Rev B 1993;48:16246-51. doi:10.1103/PhysRevB.48.16246.

[30] He C, Gao CX, Liu BG, Li M, Huang XW, Hao AM, et al. Electrical properties and phase transition of CdTe under high pressure. J Phys Condens Matter 2007;19:425223. 
doi:10.1088/0953-8984/19/42/425223.

[31] Oliver WC, Pharr GM. An improved technique for determining hardness and elastic modulus using load and displacement sensing indentation experiments. J Mater Res 1992;7:1564-83. doi:10.1557/JMR.1992.1564.

[32] Kang Z, Zhang Y, Menkara H, Wagner BK, Summers CJ, Lawrence W, et al. CdTe quantum dots and polymer nanocomposites for x-ray scintillation and imaging. Appl Phys Lett 2011;98:181914. doi:10.1063/1.3589366.

[33] Wang Z, Stroud D, Markworth A. Monte Carlo study of the liquid CdTe surface. Phys Rev B 1989;40:3129-32. doi:10.1103/PhysRevB.40.3129.

[34] Ward DK, Zhou XW, Wong BM, Doty FP, Zimmerman J a. Analytical bond-order potential for the cadmium telluride binary system. Phys Rev B - Condens Matter Mater Phys 2012;85:1-19. doi:10.1103/PhysRevB.85.115206.

[35] LAMMPS n.d. http://lammps.sandia.gov/.

[36] Vogl P, Hjalmarson HP, Dow JD. A Semi-empirical tight-binding theory of the electronic structure of semiconductors. J Phys Chem Solids 1983;44:365-78.

doi:10.1016/0022-3697(83)90064-1.

[37] Kobayashi A, Sankey OF, Dow JD. Chemical trends for defect energy levels in $\mathrm{Hg}(1-\mathrm{x}) \mathrm{CdxTe}$. Phys Rev B 1982;25:6367-79. doi:10.1103/PhysRevB.25.6367.

[38] Pérez-Conde J, Bhattacharjee a. K. Electronic structure of CdTe nanocrystals: A tight-binding study. Solid State Commun 1999;110:259-64.

doi:10.1016/S0038-1098(99)00064-2.

[39] Klimeck G, Ahmed SS, Kharche N, Korkusinski M, Usman M, Prada M, et al. Atomistic simulation of realistically sized nanodevices using NEMO 3-D-Part II: Applications. IEEE Trans Electron Devices 2007;54:2090-9. doi:10.1109/TED.2007.904877.

[40] Eshelby JD. The Determination of the Elastic Field of an Ellipsoidal Inclusion, and Related Problems. Proc R Soc A Math Phys Eng Sci 1957;241:376-96. doi:10.1098/rspa.1957.0133.

[41] Duan HL, Wang J, Huang ZP, Karihaloo BL. Eshelby formalism for nano-inhomogeneities. Proc R Soc A Math Phys Eng Sci 2005;461:3335-53. doi:10.1098/rspa.2005.1520.

[42] Hoang TL, Arsenlis A, Lee-Voigt HJ, Chrzan DC, Wirth BD. Atomistic study of Eshelby's inclusion and inhomogeneity problems in a model bec crystal. Model Simul Mater Sci Eng 2011;19:85001. doi:10.1088/0965-0393/19/8/085001.

[43] Mura T. Micromechanics of defects in solids. vol. 3. Dordrecht: Springer Netherlands; 1987. doi:10.1007/978-94-009-3489-4.

[44] Merad AE, Kanoun MB, Cibert J, Aourag H, Merad G. Stress-dependence tight binding study of tellurium-based II-VI semiconductors. Phys Lett A 2003;315:143-9. doi:10.1016/S0375-9601(03)00974-5.

[45] Freire PTC, Araújo Silva MA, Reynoso VCS, Vaz AR, Lemos V. Pressure Raman scattering of CdTe quantum dots. Phys Rev B 1997;55:6743-6. doi:10.1103/PhysRevB.55.6743.

[46] Smith PL, Martin JE. The high-pressure structures of cadmium sulphide and cadmium telluride. 
Phys Lett 1963;6:42. doi:10.1016/0031-9163(63)90214-2.

[47] Moreira SGC, da Silva EC, Mansanares AM, Barbosa LC, Cesar CL. Temperature induced stress phase transition in CdTe quantum dots observed by dielectric constant and thermal diffusivity measurements. Appl Phys Lett 2007;91:21101. doi:10.1063/1.2751128.

[48] Wu F, Zaug JM, Young CE, Zhang JZ. Pressure-induced phase transition in thiol-capped CdTe nanoparticles. J Nanosci Nanotechnol 2008;8:6528-32. doi:10.1166/jnn.2008.011.

[49] Lin Y. Water-soluble CdTe nanocrystals under high pressure. Proc. SPIE, vol. 9373, 2015, p. 93730L. doi:10.1117/12.2079540. 


\section{Figure Captions}

Figure 1. (a) Unit cell in a CdTe QD nanocomposite film; (b) loading and boundary conditions of the unit cell; (c) finite element discretization of the unit cell ( $L=64.3$ $\mathrm{nm})$.

Figure 2. (a) Zinc-blende lattice structure of CdTe; (b) atomistic configuration of a CdTe QD; (c) application of displacement field from FEM calculations to atomic sites of the QD as boundary condition.

Figure 3. (a) Distribution of the normalized stress component in the loading direction $\left(\sigma_{z} / P_{z}\right)$ inside the unit cell consisting of a CdTe QD and matrix material. (b) Variation of the normalized stress component $\left(\sigma_{z} / P_{z}\right)$ inside the QD with Young's modulus and Poisson's ratio of matrix material.

Figure 4. Strain-dependent blueshift of CdTe QDs under compressive deformation with different strain triaxiality values.

Figure 5. Energies of the lowest unoccupied states of conduction bands $\left(E_{L U}\right)$ and highest occupied states of the valence bands $\left(E_{H O}\right)$ as a function of strain under different loading conditions.

Figure 6. (a) Blueshift for QDs under compressive loading along different directions in the $(1 \overline{10})$ plane; (b) illustration of lattice directions and planes; (c) polar view of orientation-dependent blueshift for different loading conditions. 
Figure 7. (left) Average blueshift of QDs with different diameters as a function of compressive stress applied to QD composite; (right) 3-dimensional view of orientation-dependent blueshift for QDs under different stress states, both the color and surface profiles represent the amount of blueshift and redshift. 


\section{Tables}

Table 1. Material parameters for glass matrix and CdTe QDs

\begin{tabular}{|l|l|l|l|}
\hline Material & $E(\mathrm{GPa})$ & $v$ & $\rho\left(\mathrm{kg} / \mathrm{m}^{3}\right)$ \\
\hline $\mathrm{CdTe}$ & 52.0 & 0.41 & 5.86 \\
\hline glass & 69.3 & 0.17 & 2.20 \\
\hline
\end{tabular}


(a)

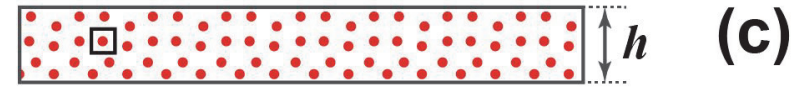

(b)

$P_{z}$
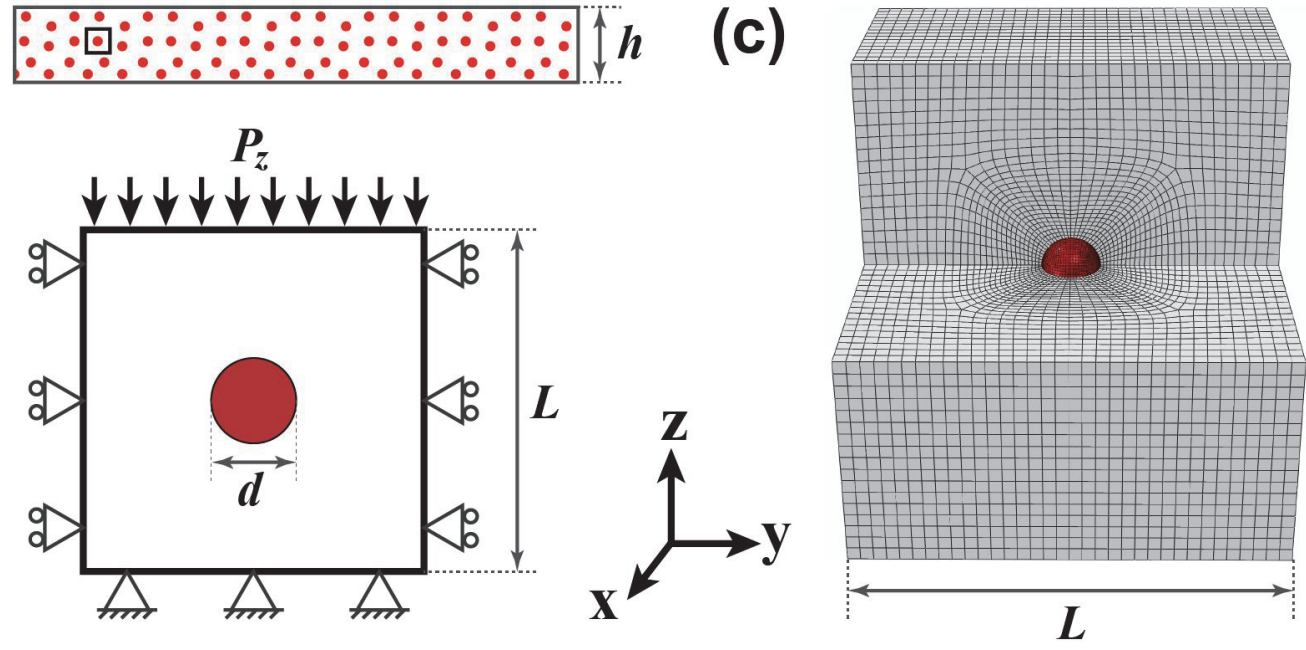

L 
(a)
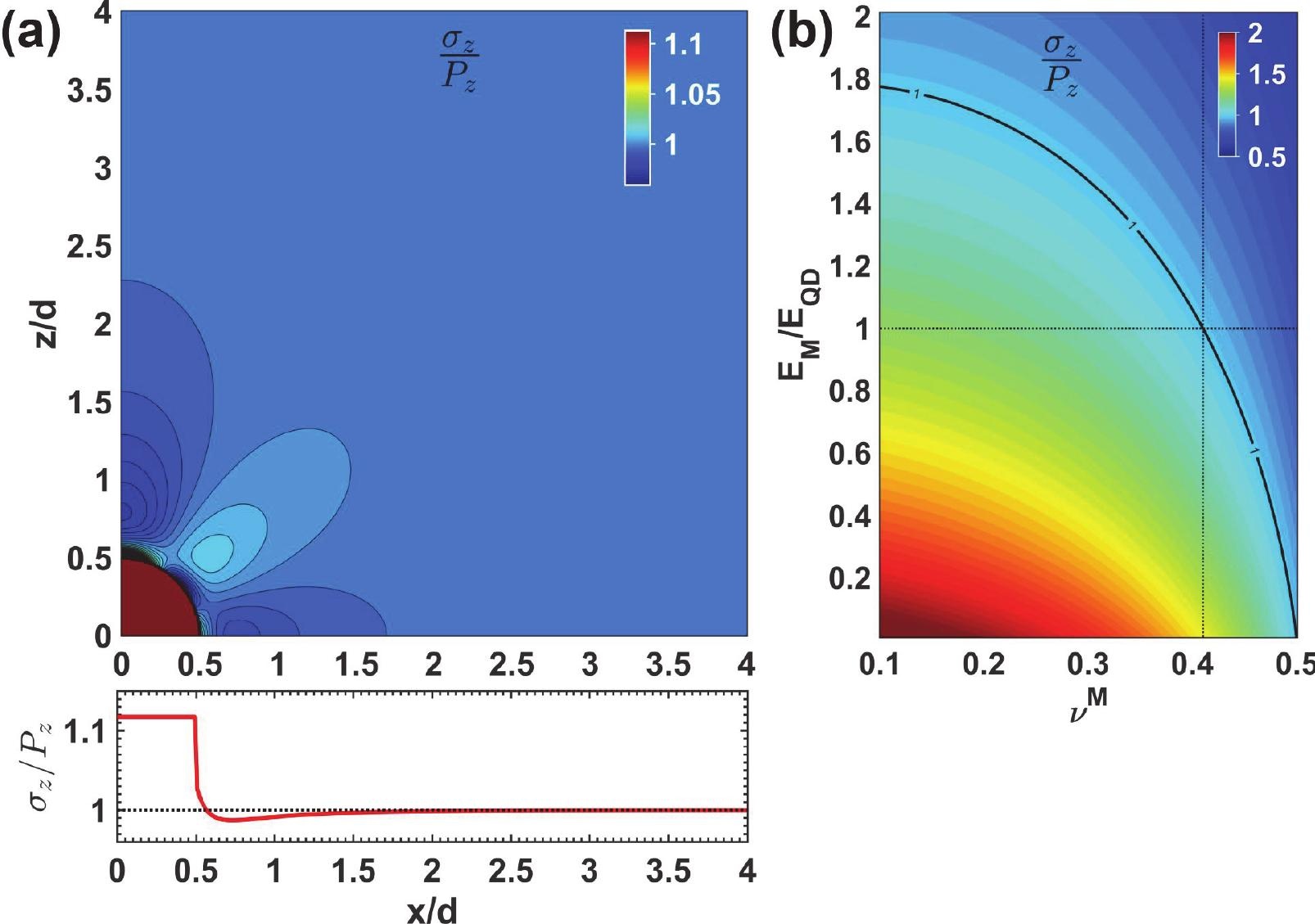
$x / d$ 


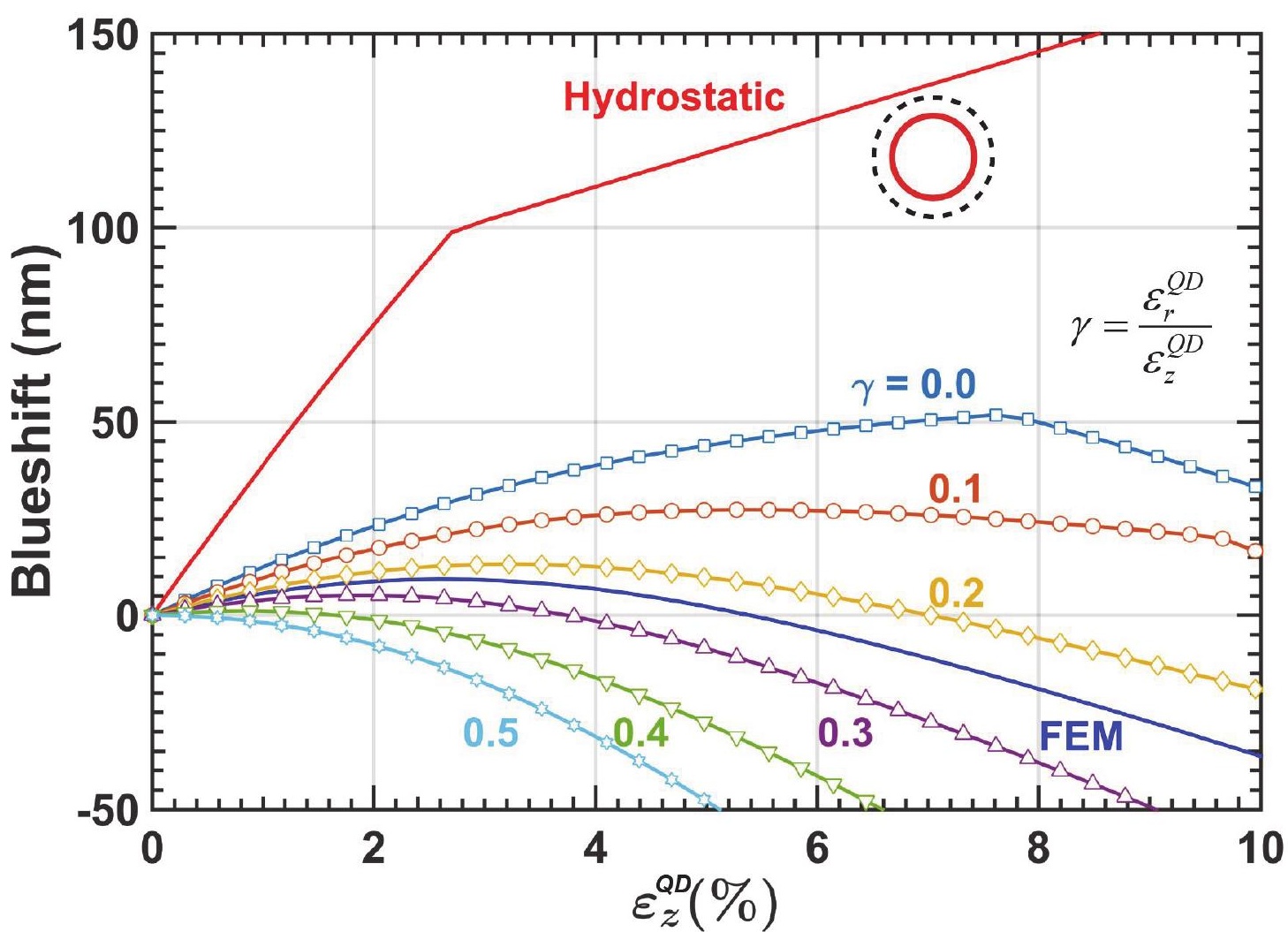




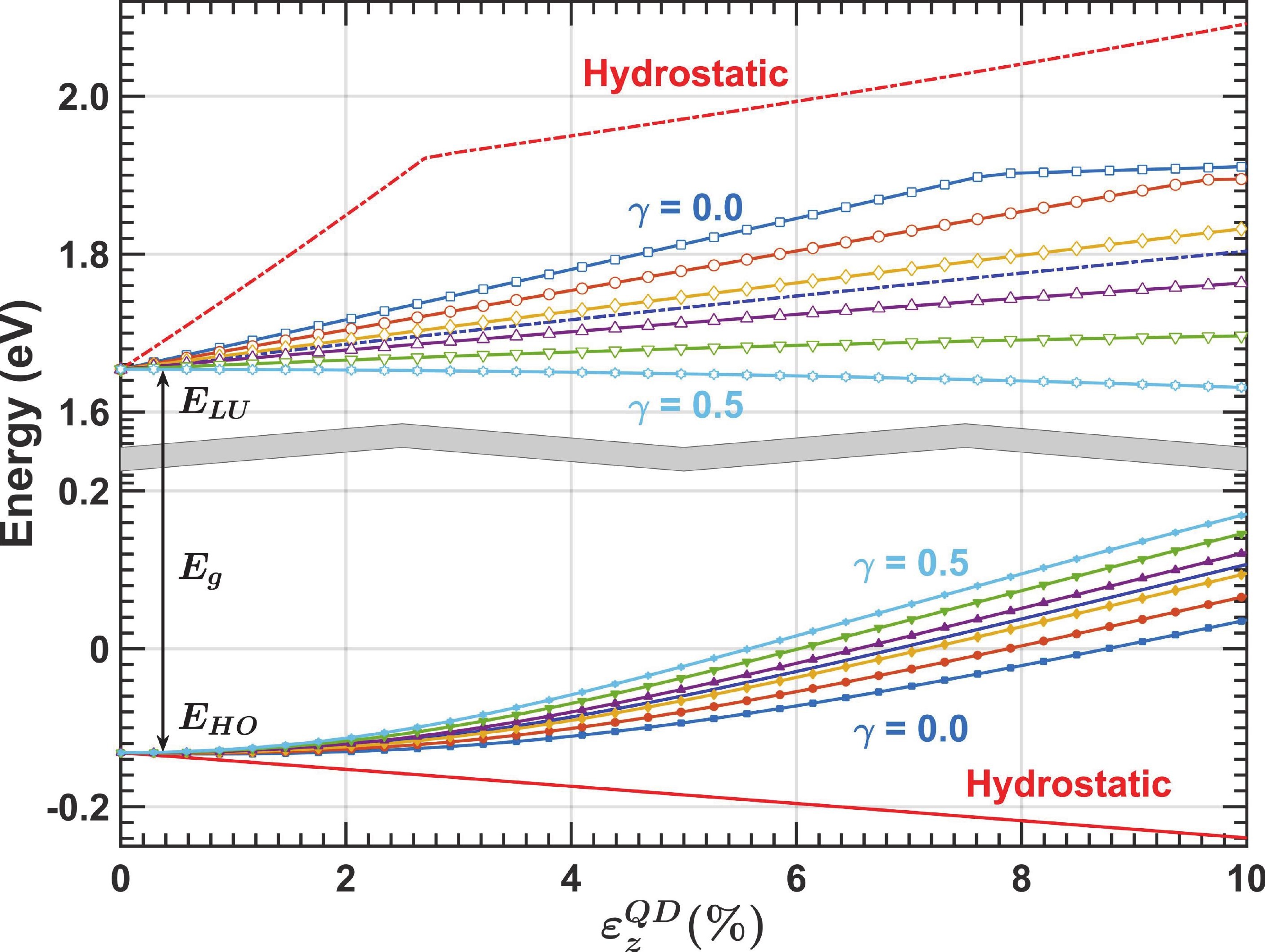


